\title{
Neck Lymph Node Boundary Levels I-IV
}

National Cancer Institute

\section{Source}

National Cancer Institute. Neck Lymph Node Boundary Levels I-IV. NCI Thesaurus. Code C142146.

A grouping of lymph nodes comprising neck lymph node boundary levels I-IV. 